\title{
APLICAÇÃO DE MODELOS MODEL-FREE E MODEL-FITTING NA CINÉTICA DA PIRÓLISE DE XISTO
}

\author{
J. P. FOLTIN ${ }^{1 *}$, A. C. L. LISBOA ${ }^{1}$ \\ ${ }^{1}$ Universidade Estadual de Campinas, Faculdade de Engenharia Química \\ e-mail: juliana.p.foltin@gmail.com
}

\begin{abstract}
RESUMO
A matéria orgânica em xisto pirobetuminoso está estreitamente relacionada com a matéria mineral, podendo ser liberada por pirólise. O xisto possui querogênio, matéria orgânica que pode ser convertida em óleo de xisto através de pirólise. Alternativas estão sendo estudadas para o petróleo, entre as quais o xisto, que por meio do processo de pirólise possibilita produzir os mesmos derivados do petróleo. Este trabalho descreve as características da cinética de pirólise de xisto da Formação Irati, fornecido pela Unidade de Negócios da Industrialização do Xisto (SIX), da PETROBRAS, localizada na cidade de São Mateus do Sul, Paraná. Termogravimetria (TG) foi utilizada para estudar o comportamento da pirólise de xisto utilizando-se temperaturas de $100{ }^{\circ} \mathrm{C}$ a $900{ }^{\circ} \mathrm{C}$. Os parâmetros cinéticos energia de ativação e fator pré-exponencial foram calculados usando métodos de Coats-Redfern, Friedman, Kissinger, Kissinger-Akahira-Sunose (KAS) e o método de Ozawa (FWO). Os resultados obtidos por cada modelo mostraram que a reação de pirólise é de primeira ordem, com valores de energias de ativação e fatores de frequência próximos aos da literatura.

Palavras-chave: cinética, model-free, pirólise, xisto.
\end{abstract}

\section{INTRODUÇÃO}

Nos últimos anos, com o aumento da demanda de recursos de energia, o xisto despontou como alternativa de energia para o petróleo. O querogênio, matéria orgânica principal do xisto, pode ser convertido em óleo de xisto através de pirólise.

Segundo DEO (2012), a análise cinética consiste em ajustar diferentes modelos de reação dados pela literatura aos dados experimentais de termogravimetria (TG). O método fornece a energia de ativação como função da conversão. $\mathrm{O}$ equipamento para a avaliação da termogravimetria é amplamente utilizado para se determinar os parâmetros cinéticos globais de pirólise. $\mathrm{O}$ equipamento é um dispositivo analítico usado para medir alterações de massa de um material submetido a uma programação de temperatura. A informação de perda de massa pode ser utilizada para construir modelos cinéticos do processo de decomposição. O método é eficiente e muito eficaz no desenvolvimento de modelos. Hubbard e Robinson (1950) mostraram que a cinética de desvolatilização pode ser explicada incluindo um período de indução térmica na análise de dados. Exige-se este período de indução térmica para se considerar os efeitos de aquecimento não isotérmicos nos experimentos realizados. Os parâmetros cinéticos estão relacionados à matéria orgânica (querogênio e betume natural). Dessa forma, as medidas dos voláteis (gás, óleo, água), betume e carbono residual são necessárias para a análise da cinética. 


\subsection{Modelos Cinéticos}

A análise cinética da decomposição se baseia na equação de Arrhenius, Equação 1, e na equação da taxa de transformação do querogênio para o produto volátil, Equação 2:

$$
\begin{gathered}
k=k_{0} \cdot e^{\left(-\frac{E}{R T}\right)} \\
\frac{d \alpha}{d t}=k(T) \cdot f(\alpha)
\end{gathered}
$$

A conversão, $\alpha$, é a forma normalizada de perda de massa da amostra e é definida de acordo com a Equação 3:

$$
a=\frac{m_{o}-m}{m_{o}-m_{f}}
$$

A combinação das Equações (1) e (2) fornece a expressão fundamental, Equação (4), em que se baseiam os métodos analíticos para calcular os parâmetros cinéticos com base em resultados de TG.

$$
\frac{d \alpha}{d t}=k_{0} \cdot f(\alpha) \cdot e^{\left(-\frac{E}{R T}\right)}
$$

A função $f(\alpha)$, Equação 5, é inserida na Equação 4, produzindo a Equação 6, em que o parâmetro $\beta=\mathrm{dT} / \mathrm{dt}$, taxa de aquecimento, foi utilizado:

$$
\begin{gathered}
f(\alpha)=(1-\alpha)^{n} \\
\frac{d \alpha}{d T}=\frac{k_{0}}{\beta} \cdot(1-\alpha)^{n} \cdot e^{\left(-\frac{E}{R T}\right)}
\end{gathered}
$$

Os métodos utilizados para calcular os parâmetros cinéticos estão apresentados na Tabela 1. Ensaios experimentais foram realizados com diferentes taxas de aquecimento. Com exceção do modelo de Coats-Redfern, relacionado ao Model-
Fitting, os outros métodos foram associados ao Model-Free.

Tabela 1 - Equações aproximadas dos métodos cinéticos.

\begin{tabular}{lccc}
\hline Método & \multicolumn{1}{c}{ Equação } & $\mathbf{X}$ & $\mathbf{Y}$ \\
\hline $\begin{array}{l}\text { Coats- } \\
\text { Redfern }\end{array}$ & $\ln \left(\frac{g(\alpha)}{T^{2}}\right)=\ln \left(\frac{k_{0} \cdot R}{\beta \cdot E}\right)-\frac{E}{R T}$ & $1000 / \mathrm{T}$ & $\ln \left(\frac{g(\alpha)}{T^{2}}\right)$ \\
Friedman & $\ln \frac{d \alpha}{d t}=\ln \left[k_{0} \cdot f(\alpha)\right]-\frac{E}{R T}$ & $1000 / \mathrm{T}$ & $\ln \left(\frac{d \alpha}{d t}\right)$ \\
FWO $\ln \left(\beta_{i}\right)=\ln \left(\frac{k_{0} \cdot E}{R \cdot g(\alpha)}\right)-5,331-1,052 \frac{E}{R T}$ & $1000 / \mathrm{T}$ & $\ln \left(\beta_{i}\right)$ \\
Kissinger & $\ln \left(\frac{\beta}{T_{m}^{2}}\right)=\ln \left(\frac{k_{0} \cdot R}{E}\right)-\frac{E}{R T_{m}}$ & $1000 / \mathrm{T}$ & $\ln \left(\frac{\beta}{T_{m}^{2}}\right)$ \\
KAS & $\ln \left(\frac{\beta}{T^{2}}\right)=\ln \left(\frac{k_{0} \cdot R}{E \cdot g(\alpha)}\right)-\frac{E}{R T}$ & $1000 / \mathrm{T}$ & $\ln \left(\frac{\beta}{T^{2}}\right)$ \\
\hline
\end{tabular}

\section{MATERIAIS E MÉTODOS}

O xisto utilizado neste trabalho foi extraído da Formação Irati e fornecido pela Petrobras/SIX, localizada na cidade de São Mateus do Sul, Paraná. A termogravimetria (TG) foi realizada com oito diferentes taxas de aquecimento: $2,5,10,15,20,25,40 \mathrm{e}$ $50{ }^{\circ} \mathrm{C} / \mathrm{min}$, desde $100{ }^{\circ} \mathrm{C}$ até $900{ }^{\circ} \mathrm{C}$ com fluxo de nitrogênio de $50 \mathrm{~mL} / \mathrm{min}$. Para a cinética de degradação, foram introduzidos em média 9,5 miligramas de cada amostra no equipamento e realizado triplicatas de cada ensaio.

\section{RESULTADOS}

A Figura 1 apresenta os dados obtidos pela TG entre $100{ }^{\circ} \mathrm{C}$ a $900{ }^{\circ} \mathrm{C}$. Observa-se a perda de massa, devida à conversão da matéria orgânica, relacionada com a temperatura: quanto maior a temperatura, maior a perda de massa. Também é possível notar o momento em que ocorrem mais significativamente as reações, entre $350{ }^{\circ} \mathrm{C}$ e $500{ }^{\circ} \mathrm{C}$. 
Figura 1 - Dados de perda de massa pela temperatura obtida pelo TG.



As Figuras 2, 3 e 4 apresentam os gráficos dos modelos de Friedman, FWO e KAS com conversões entre 0,15 e 0,65 . Foram selecionadas essas conversões, pois apresentam a taxa de conversão na faixa de temperatura em que a reação ocorre.

Os resultados obtidos a partir da termogravimetria (TG) foram elaborados de acordo com o modelo Model-Free para o cálculo dos parâmetros cinéticos. A energia de ativação $(\mathrm{E})$ e o fator pré-exponencial $\left(\mathrm{k}_{0}\right)$ foram obtidos utilizando os métodos de Friedman, KAS e FWO. Os cálculos foram feitos conforme as equações da Tabela 1. As energias de ativação (E) e o fator préexponencial $\left(\mathrm{k}_{0}\right)$ foram determinados a partir da inclinação e intercepção, respectivamente, das retas. Os resultados estão apresentados na Tabela 2.

Figura 2 - Friedman para diferentes conversões.



Figura 3 - FWO para diferentes conversões.

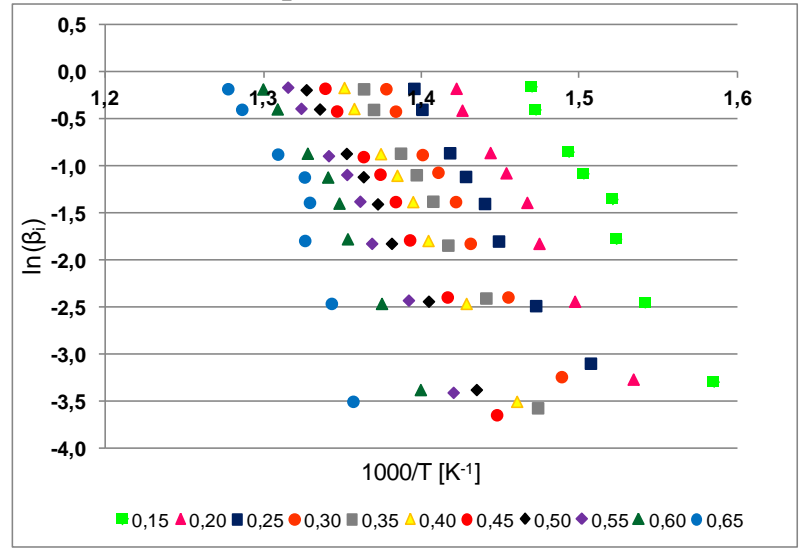

Figura 4-KAS para diferentes conversões.



Tabela 2 - Valores dos parâmetros E e $\mathrm{k}_{0}$ calculados.

\begin{tabular}{c|ccc|ccc|ccc}
\hline \multicolumn{3}{|c}{ FRIEDMAN } & \multicolumn{3}{c}{ FWO } & \multicolumn{3}{c}{ KAS } \\
\hline $\boldsymbol{\alpha}$ & $\mathbf{E}$ & $\mathbf{k}_{\mathbf{0}}$ & $\mathbf{R}^{\mathbf{2}}$ & $\mathbf{E}$ & $\mathbf{k}_{\mathbf{0}}$ & $\mathbf{R}^{\mathbf{2}}$ & $\mathbf{E}$ & $\mathbf{k}_{\mathbf{0}}$ & $\mathbf{R}^{\mathbf{2}}$ \\
\hline 0,15 & 221,26 & $8,05 \mathrm{E}+15$ & 0,9817 & 215,85 & $2,80 \mathrm{E}+17$ & 0,9772 & 216,16 & $1,72 \mathrm{E}+13$ & 0,9749 \\
0,20 & 216,56 & $2,09 \mathrm{E}+15$ & 0,9954 & 218,85 & $1,72 \mathrm{E}+17$ & 0,9926 & 218,96 & $1,02 \mathrm{E}+13$ & 0,9919 \\
0,25 & 231,76 & $2,45 \mathrm{E}+16$ & 0,9992 & 211,48 & $2,96 \mathrm{E}+16$ & 0,9899 & 211,00 & $1,58 \mathrm{E}+12$ & 0,9889 \\
0,30 & 234,04 & $3,02 \mathrm{E}+16$ & 0,9977 & 218,42 & $7,66 \mathrm{E}+16$ & 0,9958 & 218,15 & $4,25 \mathrm{E}+12$ & 0,9954 \\
0,35 & 228,07 & $9,40 \mathrm{E}+15$ & 0,9946 & 239,69 & $2,41 \mathrm{E}+18$ & 0,9954 & 240,42 & $1,58 \mathrm{E}+14$ & 0,9949 \\
0,40 & 248,73 & $2,44 \mathrm{E}+17$ & 0,9941 & 239,22 & $1,82 \mathrm{E}+18$ & 0,9978 & 239,81 & $1,17 \mathrm{E}+14$ & 0,9976 \\
0,45 & 239,65 & $4,42 \mathrm{E}+16$ & 0,9949 & 246,34 & $4,98 \mathrm{E}+18$ & 0,9915 & 247,20 & $3,34 \mathrm{E}+14$ & 0,9906 \\
0,50 & 252,95 & $2,98 \mathrm{E}+17$ & 0,9881 & 236,63 & $7,98 \mathrm{E}+17$ & 0,9967 & 236,88 & $4,83 \mathrm{E}+13$ & 0,9964 \\
0,55 & 269,49 & $2,99 \mathrm{E}+18$ & 0,9688 & 245,29 & $2,76 \mathrm{E}+18$ & 0,9924 & 245,87 & $1,76 \mathrm{E}+14$ & 0,9916 \\
0,60 & 299,70 & $2,04 \mathrm{E}+20$ & 0,9313 & 254,70 & $9,38 \mathrm{E}+18$ & 0,9824 & 255,60 & $6,30 \mathrm{E}+14$ & 0,9806 \\
0,65 & 328,18 & $4,56 \mathrm{E}+21$ & 0,7028 & 301,11 & $9,27 \mathrm{E}+21$ & 0,8678 & 304,12 & $8,34 \mathrm{E}+17$ & 0,8580 \\
\hline Média & $\mathbf{2 5 1 , 8 5}$ & $\mathbf{4 , 3 3 + 2 0}$ & & $\mathbf{2 3 8 , 8 7}$ & $\mathbf{8 , 4 5 + 2 0}$ & & $\mathbf{2 0} 39, \mathbf{2 3}$ & $\mathbf{7 , 6 0 + 1 6}$
\end{tabular}

Verifica-se que, para os três métodos, há um aumento da energia de ativação (E) em função da conversão $(\alpha)$, com um 
comportamento anômalo entre 0,35 e 0,45. Comparando os valores médios nota-se que os resultados de FWO e KAS são muito semelhantes, enquanto que os de Friedman destoam dos demais. Para o fator préexponencial $\left(\mathrm{k}_{0}\right)$, verifica-se que seu valor varia bastante com o aumento da taxa de conversão. Comparando os valores médios observa-se que os resultados de FWO e Friedman possuem a mesma ordem de grandeza, enquanto que os de KAS desviam dos demais.

A Figura 5 apresenta o gráfico de Coats-Redfern, calculado a partir da equação de Model-Fitting fornecida pela Tabela 1. Graficamente, o método de CoatsRedfern apresenta um comportamento linear dos dados obtidos. Os resultados de energia de ativação (E) e fator préexponencial $\left(\mathrm{k}_{0}\right)$ são menores que os dos outros métodos, como pode ser visto na Tabela 3. Observa-se também que para este modelo e para uma mesma taxa de conversão $(\alpha)$, ocorre um comportamento ascendente enquanto que para os demais o comportamento é descendente.

$\mathrm{O}$ modelo de Kissinger mostrado na Figura 6 utiliza a mesma metodologia dos modelos de Friedman, FWO e KAS. O que difere é que Kissinger utiliza a temperatura máxima do pico da perda de massa. $\mathrm{Na}$ Tabela 4 estão os dados calculados a partir da equação do modelo de Kissinger. É visível pelo gráfico um comportamento linear em que os resultados são calculados a partir das diferentes taxas de aquecimento.
Figura 5 - Coats-Redfern para diferentes conversões.



Tabela 3 - Parâmetros cinéticos calculados pelo método de Coats-Redfern.

\begin{tabular}{cccc}
\hline $\boldsymbol{\beta}$ & $\mathbf{E}$ & $\mathbf{k}_{\mathbf{0}}$ & $\mathbf{R}^{\mathbf{2}}$ \\
\hline 2 & 65,51 & $1,63 \mathrm{E}+00$ & 0,9602 \\
5 & 75,41 & $1,94 \mathrm{E}+01$ & 0,9760 \\
10 & 75,50 & $3,12 \mathrm{E}+01$ & 0,9780 \\
15 & 76,29 & $5,02 \mathrm{E}+01$ & 0,9730 \\
20 & 82,58 & $1,87 \mathrm{E}+02$ & 0,9761 \\
25 & 79,82 & $1,25 \mathrm{E}+02$ & 0,9777 \\
40 & 79,74 & $1,61 \mathrm{E}+02$ & 0,9802 \\
50 & 76,19 & $1,00 \mathrm{E}+02$ & 0,9823 \\
\hline
\end{tabular}

Figura 6 - Método de Kissinger para diferentes taxas de aquecimento.



Tabela 4 - Parâmetros cinéticos calculados pelo método de Kissinger.

\begin{tabular}{ccc}
\hline $\mathbf{E}$ & $\mathbf{k}_{\mathbf{0}}$ & $\mathbf{R}^{\mathbf{2}}$ \\
\hline 199,65 & $1,50 \mathrm{E}+11$ & 0,9769 \\
\hline
\end{tabular}

A Figura 7 apresenta a energia de ativação em função conversão $(\alpha)$ para os 
diversos métodos avaliados. Verifica-se que, para os métodos de Friedman, FWO e KAS, os dados obedecem à curvas polinomiais de segunda ordem cujas constantes estão dadas na Tabela 5. Para os métodos de Kissinger e Coats-Redfern não foram inclusos os dados no gráfico pelo comportamento dos dados ser linear.

Figura 7 - Energia de ativação em função da conversão.

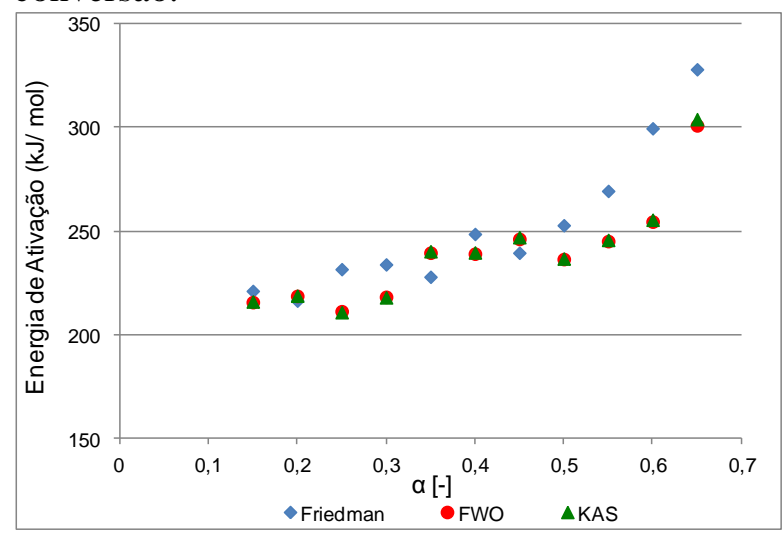

Tabela 5 - Regressão polinomial de segunda ordem para os métodos de Friedman, FWO e KAS.

\begin{tabular}{ccccc}
\hline Método & $\mathbf{x}_{\mathbf{2}}$ & $\mathbf{x}_{\mathbf{1}}$ & $\mathbf{x}_{\mathbf{0}}$ & $\mathbf{R}^{\mathbf{2}}$ \\
\hline Friedman & 568,29 & $-267,4$ & 253,68 & 0,9434 \\
FWO & 299,35 & $-109,63$ & 227,34 & 0,8089 \\
KAS & 315,99 & $-119,12$ & 228,66 & 0,8041 \\
\hline
\end{tabular}

Os resultados obtidos são semelhantes aos obtidos por BAI e GUO (2015), SLOPIECKA et. al. (2012) e WANG et. al. (2013).

\section{CONCLUSÃO}

Os resultados experimentais, obtidos com oito taxas de aquecimento, possibilitaram a obtenção de parâmetros cinéticos para a pirólise de xisto através do método Model-Free, com valores médios próximos entre os modelos KAS e FWO. Com relação ao Model-Fitting, os resultados obtidos estão uma ordem de grandeza diferente quando comparados aos do Model- Free.

\section{NOMENCLATURA}

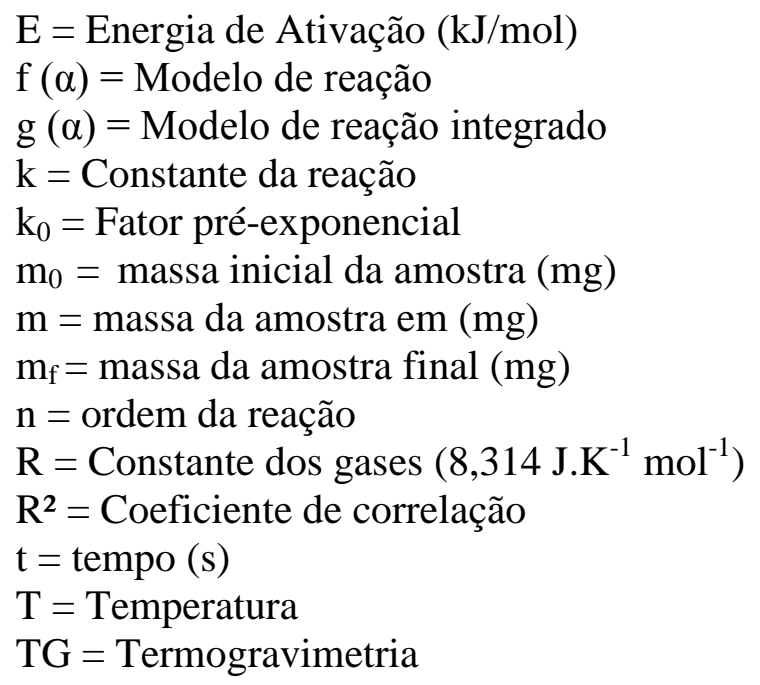

\section{Símbolos gregos}

$\alpha=$ Extensão da reação

$\beta=$ Taxa de aquecimento

\section{REFERÊNCIAS}

BAI, F., GUO W, et al, Kinetic study on the pyrolysis behavior of Huadian oil shale via non-isothermal termogravimetric data, Elsevier, v. 146, p. 111-118, 2015.

DEO M., TIWARI P., Compositional and kinetic analysis of oil shale pyrolysis using TGA-M. Fuel. v. 94, p. 333-34, 2012.

HUBBARD, A B., ROBINSON, W. E., A Thermal Decomposition Study of Colorado Oil Shale, USA Bureal of Mines Report of investigations v. 4744, p. 24, 1950.

SLOPIECKA, K., BARTOCCI, P., FANTOZZI, F., Thermogravimetric analysis and kinetic study of poplar wood pyrolysis, Elsevier, v. 97, p. 491-497, 2012.

WANG, H., WANG Z., DENG S. et al, Pyrolysis kinetic study of Huadian oil shale, 
spent oil shale and their mixtures by Thermogravimetric Analysis, Elsevier v. 110, p. 103-108, 2013.

\section{AGRADECIMENTOS}

Pelo apoio financeiro do Conselho Nacional de Pesquisa e Desenvolvimento CNPq, ao professor Arno de Klerk da Universidade de Alberta, e à Universidade Estadual de Campinas. 